ESAIM: M2AN 47 (2013) 1533-1552

DOI: $10.1051 / \mathrm{m} 2 \mathrm{an} / 2013079$
ESAIM: Mathematical Modelling and Numerical Analysis

www.esaim-m2an.org

\title{
FIRST ORDER SECOND MOMENT ANALYSIS FOR STOCHASTIC INTERFACE PROBLEMS BASED ON LOW-RANK APPROXIMATION*
}

\author{
Helmut HARBRECht ${ }^{1}$ AND Jingzhi LI $^{2}$
}

\begin{abstract}
In this paper, we propose a numerical method to solve stochastic elliptic interface problems with random interfaces. Shape calculus is first employed to derive the shape-Taylor expansion in the framework of the asymptotic perturbation approach. Given the mean field and the two-point correlation function of the random interface, we can thus quantify the mean field and the variance of the random solution in terms of certain orders of the perturbation amplitude by solving a deterministic elliptic interface problem and its tensorized counterpart with respect to the reference interface. Error estimates are derived for the interface-resolved finite element approximation in both, the physical and the stochastic dimension. In particular, a fast finite difference scheme is proposed to compute the variance of random solutions by using a low-rank approximation based on the pivoted Cholesky decomposition. Numerical experiments are presented to validate and quantify the method.
\end{abstract}

Mathematics Subject Classification. 60H15, 60H35, 65C20, 65C30.

Received May 4, 2011. Revised December 13, 2012.

Published online August 14, 2013.

\section{INTRODUCTION}

This paper is devoted to an efficient numerical method for physical problems governed by elliptic partial differential equations (PDEs) with discontinuous coefficients across random interfaces, namely stochastic elliptic interface problems (SEIP). A distinct feature of elliptic interface problems (EIPs) lies in the fact that their solutions have high regularity on each individual material region but low global regularity due to the discontinuity of the material coefficients across the interface. Many numerical methods such as finite difference methods, finite element methods, and finite volume methods have been developed in the past few decades for deterministic elliptic interface problems. We refer the reader to the monograph [32] and the references therein.

Here, we will model and solve EIPs which involve new challenges by random interfaces. This is of immense interest in the computational simulation in nano-physics, biology and chemistry, where one has unsharp interfaces like rough cross sections, cell membranes and molecular surfaces for instance. We emphasize that traditional

\footnotetext{
Keywords and phrases. Elliptic interface problem, stochastic interface, low-rank approximation, pivoted Cholesky decomposition.

* The research of HH has been supported by the Swiss National Fond under project "Rapid solution of boundary value problems on stochastic domains". The research of JL (correspondence author) has been supported by the NSF of China (No. 11201453 and 91130022).

1 Helmut Harbrecht, Mathematisches Institut, Universität Basel, Rheinsprung 21, 4051 Basel, Switzerland. helmut.harbrecht@unibas.ch

2 Faculty of Science, South University of Science and Technology of China, Shenzhen 518055, P. R. China. li.jz@sustc.edu.cn
} 
methods for PDEs with random coefficients fail since the underlying statistics is inherently non-smooth. Hence, we shall use techniques which have been developed in the past five years for PDEs with an uncertain computational domain, see, e.g., $[9,20,23,44]$ and the references therein. Broadly speaking, there are two categories of approaches to solve stochastic PDE.

(A) Monte Carlo methods (MC) (see [29,35] and the references therein): This is the most general methodology to treat randomness by sampling numerous draws of the random input data according to some a priorily known or empirical distribution, where each draw entails the computation of a deterministic PDE. Then, the statistics like the mean and variance of these samples is formed. By interpreting the realizations as draws of independent and identically distributed (i.i.d.) random variables, one can show that the convergence rate of MC methods is inversely proportional to the square root of the number of samples. Dimension-independence is the key advantage of MC methods. Nevertheless, to guarantee a certain accuracy, a huge number of samples has to be evaluated which could require formidable computing resources. Recently, the efficient multilevel MC method has been proposed to solve elliptic PDEs with stochastic coefficients in asymptotically optimal complexity [5].

(B) Deterministic approaches: If the statistical description of the random input data is specified, one can mathematically characterize the uncertainty in the input data and recast the original stochastic PDE into a deterministic one. From this, the statistics of the unknown random field is derived. There are two subclasses in this case.

(B.1) Perturbation methods $[1,19,20,22,27,28]$ : The pivotal idea of the perturbation approach for stochastic PDEs is the expansion of the random field at hand around the related input parameter's expectation via a Taylor series. This is applicable if the perturbation amplitude is small. Then, the expansion is substituted into the original problem. Equating the terms of equal order in the asymptotic expansion of the output parameter, one arrives at the corresponding zeroth-, first- and second-order subproblems, etc.

(B.2) Stochastic Galerkin methods $[2-4,12,13,16,17,33,36,37,40,41,43]$ : The rationale behind these methods is to express, through the decomposition of physical and stochastic variables (e.g. by the Karhunen-Loève expansion), the random field as the tensor product of functions in the physical domain and random variables in the stochastic domain. Then one adopts, respectively, finite element spaces for the physical approximation and wavelets or (generalized) chaos polynomials for the stochastic approximation. Efficient solvers are achieved by employing sparse grid techniques to mitigate the curse of dimensionality.

In the present paper, we will employ the perturbation approach to treat the SEIP. Due to the uncertainty of the interface, the solution of the elliptic system itself becomes a random field. The statistics of the random solution is obtained by transforming the stochastic problem into a couple of deterministic ones (possibly) in high dimensions. It is common sense that the knowledge of the complete statistics of the input random field is rarely known in practice. Therefore, as in [23], we impose two additional assumptions for the model in the current work. Namely, (i) that the perturbation amplitude of the random interface around its mean is relatively small and (ii) that the mean field and the two-point correlation function of the random interface are known empirically or from elsewhere. It is emphasized that the underlying stochastic process needs not to be explicitly known. More precisely, the SEIP may well be reformulated as follows: Given the statistics of the interface's random perturbation, how to compute the statistics of the SEIP's random solution?

The use of shape sensitivity analysis offers the possibility to treat such problems, which, to our opinion, is not known and new to the mathematical and engineering community. The novelty of our approach lies in the derivation of a tensorized PDE for the two-point correlation function of the shape derivative. This PDE constitutes the basis to derive the variance of random solutions. It is worth noting that solving such a tensorized PDE is a highly-nontrivial task with challenging demands if one resorts to sparse grid techniques. As an alternative approach, we use the pivoted Cholesky decomposition to solve this equation. It is worth remarking that the Monte Carlo approach employs a great number of sample interfaces to solve deterministic EIPs with each sample interface, which is a demanding job, not to mention the work about remeshing for each sample interface to avoid the sub-optimal convergence [31]. 
We mention that an approach which is similar to ours was first utilized and analyzed in [1] to address elliptic PDEs with stochastic coefficients. However, in difference to [1], we also derive the asymptotic error of the variance. Moreover, in [11], which is a more recent work than ours, a very general and abstract framework of the present first order perturbation approach has been formulated. It covers even nonlinear operator equations with random input data and particularly includes also random domains. Besides precise estimates on the constants which are involved in the asymptotic expansions, also expressions for the random solution's higher order moments are derived by multilinear, tensorized operator equations. Nevertheless, we focus here on the specific application to random interface problems and the related numerical realization.

The rest of the paper is organized as follows. In Section 2, we set up a SEIP model and mathematically describe the randomness and the statistics of stochastic interfaces. In Section 3, we derive the shape derivative of solutions to EIPs via shape calculus. In Section 4, we formulate a deterministic elliptic interface problem and its tensorized counterpart for the mean and two-point correlation of the random solution, respectively. In Section 5, error estimates for the fully discrete approximation of the mean field and two-point correlation function are derived in both stochastic and physical dimensions. A fast algorithm based on the low-rank approximation via the pivoted Cholesky decomposition is proposed as an efficient alternative to compute the two-point correlation function of random solutions. In Section 6, numerical experiments are shown to compare our proposed deterministic implementation with the Monte Carlo method and to demonstrate promising advantages.

\section{Mathematical Formulation}

\subsection{Model problem}

Consider the following EIP arising from the heat conduction model in physics:

$$
\begin{aligned}
-\nabla \cdot(\alpha(\mathbf{x}, \omega) \nabla u(\mathbf{x}, \omega)) & =f(\mathbf{x}) & & \text { in } D^{-}(\omega) \cup D^{+}(\omega), \\
{[u(\mathbf{x}, \omega)] } & =0 & & \text { on } \Gamma(\omega), \\
{\left[\alpha(\mathbf{x}, \omega) \frac{\partial u}{\partial \mathbf{n}}(\mathbf{x}, \omega)\right] } & =0 & & \text { on } \Gamma(\omega), \\
u(\mathbf{x}, \omega) & =0 & & \text { on } \partial D,
\end{aligned}
$$

where $D \subset \mathbb{R}^{d}, d=2,3$, is assumed to be a simply-connected and convex domain with Lipschitz boundary $\partial D$. The domain $D$ is occupied by two random subdomains $D^{-}(\omega)$ and $D^{+}(\omega)$ of different materials, where $D^{-}(\omega) \Subset D, D^{+}(\omega):=D \backslash \overline{D^{-}(\omega)}$. These two subdomains are separated by the random interface $\Gamma(\omega):=$ $\partial D^{-}(\omega)$ (see Fig. 1). Equations (2.2) and (2.3) are the jump conditions which the solution $u(\mathbf{x}, \omega)$ has to meet on the random interface $\Gamma(\omega)$. The function $f(\mathbf{x})$ is a deterministic source term satisfying $f \in C^{\infty}(\bar{D})$. Moreover, $\mathbf{n}(\mathbf{x}, \omega)$ denotes the unit normal vector to the interface $\Gamma(\omega)$, pointing to the interior of $D^{+}(\omega)$. As regards the smoothness of the random interface, we have made the following assumption:

Assumption 2.1. The interface $\Gamma(\omega)$ is $C^{2,1}$-smooth.

Note that a boundary $\Gamma$ (or the domain with boundary $\Gamma$ ) is $C^{k, 1}$-smooth if there exist for all $\mathbf{x} \in \Gamma$ a neighborhood $U(\mathbf{x}) \subset \Gamma$ and an associated diffeomorphism $\gamma:(0,1)^{d-1} \rightarrow U(\mathbf{x})$ such that $\gamma \in C^{k, 1}\left((0,1)^{d-1}, \mathbb{R}^{d}\right)$, see [42] for example.

For any arbitrarily fixed realization $\omega$ of a continuous function $u(\cdot, \omega)$ we denote by $u^{-}(\cdot, \omega)$ (resp. $\left.u^{+}(\cdot, \omega)\right)$ its restriction onto $D^{-}(\omega)$ (resp. $D^{+}(\omega)$ ). Throughout the paper, the jump $[u(\cdot, \omega)]$ is always understood to be $u^{-}(\cdot, \omega)-u^{+}(\cdot, \omega)$ on $\Gamma(\omega)$ in the sense of the trace for each sample $\omega$. The random coefficient field $\alpha(\mathbf{x}, \omega)$, due to the uncertainty of the interface $\Gamma(\omega)$, is assumed to be a piecewise smooth function, namely

$$
\alpha(\mathbf{x}, \omega)=\beta(\mathbf{x})+\chi_{D^{-}(\omega)}(\mathbf{x}) \gamma(\mathbf{x}) \text { in } D
$$




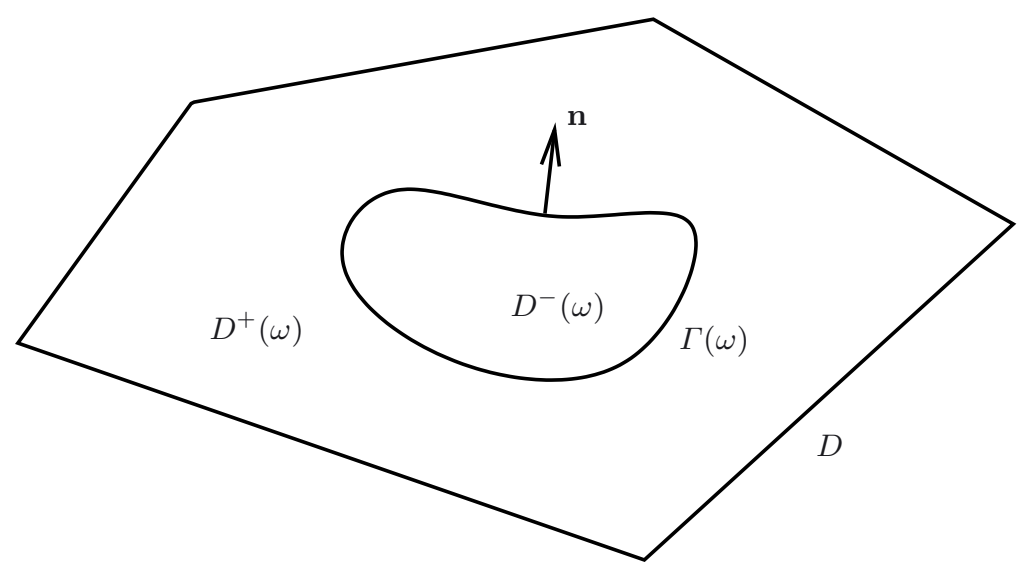

FiguRE 1. Domain sketch with stochastic interface.

with smooth (deterministic) functions $\beta(\mathbf{x})$ and $\gamma(\mathbf{x})$. The function $\chi_{D^{-}(\omega)}(\mathbf{x})$ is the characteristic function of the random subdomain $D^{-}(\omega)$, i.e., it is 1 if $\mathbf{x} \in D^{-}(\omega)$ and 0 otherwise. Moreover, we denote $\alpha^{-}(\mathbf{x}, \omega)$ (resp. $\left.\alpha^{+}(\mathbf{x}, \omega)\right)$ the restriction of $\alpha(\mathbf{x}, \omega)$ onto $D^{-}(\omega)$ (resp. $D^{+}(\omega)$ ). Hence, the flux jump satisfies

$$
\left[\alpha(\omega) \frac{\partial u}{\partial \mathbf{n}}(\omega)\right]:=\alpha^{-} \frac{\partial u^{-}}{\partial \mathbf{n}}(\omega)-\alpha^{+} \frac{\partial u^{+}}{\partial \mathbf{n}}(\omega) \quad \text { on } \Gamma(\omega) .
$$

\subsection{Stochastic interfaces}

To address the randomness of the interface, it is of first priority to model the stochastic interface in a mathematical way.

Fix any fixed reference interface $\Gamma \in C^{3,1} \subset C^{2,1}$, which is a closed and orientable manifold of co-dimension one, separating two fixed subdomains $D^{-}$and $D^{+}$. Let $\mathbf{n}(\mathbf{x})$ denote the unit normal vector along $\Gamma$ pointing into $D^{+}$. From $\Gamma \in C^{3,1}$, we conclude $\mathbf{n}(\mathbf{x}) \in C^{2,1}\left(\Gamma, \mathbb{R}^{d}\right)$. Thus, for any

$$
\kappa \in \mathscr{A}:=\left\{\nu \in C^{2,1}(\Gamma, \mathbb{R}):\|\nu\|_{C^{2,1}(\Gamma, \mathbb{R})} \leq 1\right\},
$$

we obtain a normal variation of the interface

$$
\mathbf{V}(\mathbf{x}):=\kappa(\mathbf{x}) \mathbf{n}(\mathbf{x}) \in C^{2,1}\left(\Gamma, \mathbb{R}^{d}\right),
$$

cf. [14,34]. It exists an $\varepsilon_{0}>0$, such that for any $0 \leq \varepsilon \leq \varepsilon_{0}$ the perturbed interface

$$
\Gamma_{\varepsilon}:=\{\mathbf{x}+\varepsilon \kappa(\mathbf{x}) \mathbf{n}(\mathbf{x}): \mathbf{x} \in \Gamma\} \in C^{2,1}
$$

is well defined. In particular, $\Gamma_{\varepsilon}$ determines uniquely the subdomains $D_{\varepsilon}^{-}$and thus $D_{\varepsilon}^{+}:=D \backslash \overline{D_{\varepsilon}^{-}}$. Here the second assumption on interfaces comes into play to facilitate the perturbation analysis.

Assumption 2.2. The upper bound $\varepsilon_{0}$ is sufficiently small to ensure that the interface $\Gamma_{\varepsilon}$ is not degenerate and lies still inside the domain $D$.

To account for the randomness of stochastic interfaces, it is equivalent to describe mathematically the random field

$$
\kappa(\mathbf{x}, \omega): \Gamma \times \Omega \rightarrow \mathbb{R} .
$$

The stochastic interfaces $\Gamma(\omega)$ can be rigorously characterized by a complete probability space $(\Omega, \Sigma, P)$ where $\Omega$ is the set of all sample draws, namely realizations $\omega \mapsto \kappa(\cdot, \omega) \in \mathscr{A}, \Sigma$ is the Borel $\sigma$-algebra of $\mathscr{A}$, and 
$P: \Sigma \rightarrow[0,1]$ is a probability measure on the measurable space $(\Omega, \Sigma)$. For probability spaces on Banach spaces see $[6,30]$ and references therein.

Instead of grasping full knowledge of the random solutions, we are more interested in the statistics of the random field, $u: D \times \Omega \rightarrow \mathbb{R}$ which satisfies the PDE (2.1)-(2.4) $P$-a.s. in $\Omega$.

\subsection{Mean and two-point correlation}

Throughout the rest of this work, the first and second moments of the $P$-measurable mapping $\kappa(\mathbf{x}, \omega): \Omega \rightarrow \mathscr{A}$ have to be given. Thus we make the last assumption as follows:

Assumption 2.3. The random field $\kappa(\mathbf{x}, \omega)$ has a finite second moment with respect to $P$ which belongs to the Bochner space $L^{2}\left(\Omega, C^{2,1}(\Gamma, \mathbb{R})\right)$.

Given a random field $\kappa \in L^{2}\left(\Omega, C^{2,1}(\Gamma, \mathbb{R})\right)$ and a perturbation amplitude $\varepsilon$ with $0 \leq \varepsilon \leq \varepsilon_{0}$, the random interface can be parametrized by

$$
\Phi_{\varepsilon}:\left\{\begin{array}{l}
\Gamma \times \Omega \rightarrow \mathbb{R}^{d}, \\
(\mathbf{x}, \omega) \mapsto \mathbf{x}+\varepsilon \kappa(\mathbf{x}, \omega) \mathbf{n}(\mathbf{x}) .
\end{array}\right.
$$

A realization of the subdomains $D_{\varepsilon}^{ \pm}(\omega)$ is thus separated by the interface

$$
\Gamma_{\varepsilon}(\omega):=\left\{\Phi_{\varepsilon}(\mathbf{x}, \omega): \mathbf{x} \in \Gamma\right\}, \quad \omega \in \Omega .
$$

Due to $\kappa \in L^{2}\left(\Omega, C^{2,1}(\Gamma, \mathbb{R})\right)$, the first two statistical moments of the random interface variation $\kappa(\mathbf{x}, \omega)$ are pointwise finite. They are defined as follows: the mean

$$
\mathbb{E}_{\kappa}(\mathbf{x}):=\int_{\Omega} \kappa(\mathbf{x}, \omega) \mathrm{d} P(\omega)=\mathbb{E}(\kappa(\mathbf{x}, \omega)), \quad \mathbf{x} \in \Gamma,
$$

the two-point correlation function

$$
\operatorname{Cor}_{\kappa}(\mathbf{x}, \mathbf{y}):=\int_{\Omega} \kappa(\mathbf{x}, \omega) \kappa(\mathbf{y}, \omega) \mathrm{d} P(\omega)=\mathbb{E}(\kappa(\mathbf{x}, \omega) \kappa(\mathbf{y}, \omega)), \quad \mathbf{x}, \mathbf{y} \in \Gamma
$$

and the covariance

$$
\operatorname{Covar}_{\kappa}(\mathbf{x}, \mathbf{y}):=\operatorname{Cor}_{\kappa}(\mathbf{x}, \mathbf{y})-\mathbb{E}_{\kappa}(\mathbf{x}) \mathbb{E}_{\kappa}(\mathbf{y}), \quad \mathbf{x}, \mathbf{y} \in \Gamma .
$$

Here, by $\mathbb{E}$ we denote the expectation or ensemble average with respect to the probability measure $P$.

It obviously holds that

$$
\mathbb{E}\left(\Gamma_{\varepsilon}(\omega)\right):=\left\{\mathbb{E}\left(\Phi_{\varepsilon}(\mathbf{x}, \omega)\right), \mathbf{x} \in \Gamma\right\}=\left\{\mathbf{x}+\varepsilon \mathbb{E}_{\kappa}(\mathbf{x}) \mathbf{n}(\mathbf{x}), \mathbf{x} \in \Gamma\right\} .
$$

Without loss of generality, we may assume that the random field $\kappa(\mathbf{x}, \omega)$ is centered, namely

$$
\mathbb{E}_{\kappa}(\mathbf{x})=0 .
$$

Otherwise, we may readjust the reference interface such that (2.5) holds. Therefore, $\mathbb{E}\left(\Gamma_{\varepsilon}(\omega)\right)=\Gamma$ and

$$
\operatorname{Covar}_{\kappa}(\mathbf{x}, \mathbf{y})=\operatorname{Cor}_{\kappa}(\mathbf{x}, \mathbf{y}) .
$$

Furthermore, once $\mathbb{E}_{\kappa}(\mathbf{x})$ and $\operatorname{Cor}_{\kappa}(\mathbf{x}, \mathbf{y})$ are given, it holds

$$
\begin{aligned}
\operatorname{Cor}_{\Phi_{\varepsilon}}(\mathbf{x}, \mathbf{y}) & =\mathbb{E}\left(\Phi_{\varepsilon}(\mathbf{x}, \omega) \Phi_{\varepsilon}(\mathbf{y}, \omega)\right) \\
& =\mathbf{x} \cdot \mathbf{y}+\varepsilon^{2} \operatorname{Cor}_{\kappa}(\mathbf{x}, \mathbf{y}) \mathbf{n}(\mathbf{x}) \cdot \mathbf{n}(\mathbf{y}) .
\end{aligned}
$$

This means that the two-point correlation function of the random interface can be modeled by that of the random variation $\kappa(\mathbf{x}, \omega)$. 


\section{Shape Calculus}

In this section, we return to the deterministic world to develop the shape-Taylor expansion of solutions to EIPs with respect to the reference interface $\Gamma \in C^{3,1}$ via shape calculus. To that end, we derive the shape gradient based on the velocity method ( $c f .[24-26,34,39])$.

Consider a deterministic EIP with respect to the reference interface $\Gamma$

$$
\begin{array}{rlrl}
-\nabla \cdot(\alpha \nabla u) & =f & & \text { in } D^{-} \cup D^{+}, \\
{[u]=0} & & \text { on } \Gamma, \\
{\left[\alpha \frac{\partial u}{\partial \mathbf{n}}\right]} & =0 & & \text { on } \Gamma, \\
u & =0 & & \text { on } \partial D,
\end{array}
$$

and a deterministic perturbed PDE with respect to perturbed interface $\Gamma_{\varepsilon}$

$$
\begin{array}{rlrl}
-\nabla \cdot\left(\alpha \nabla u_{\varepsilon}\right) & =f & & \text { in } D_{\varepsilon}^{-} \cup D_{\varepsilon}^{+}, \\
{\left[u_{\varepsilon}\right]=0} & & \text { on } \Gamma_{\varepsilon}, \\
{\left[\alpha \frac{\partial u_{\varepsilon}}{\partial \mathbf{n}}\right]=0} & & \text { on } \Gamma_{\varepsilon} \\
u_{\varepsilon}=0 & & \text { on } \partial D
\end{array}
$$

where $\Gamma_{\varepsilon}:=\{\mathbf{x}+\varepsilon \kappa(\mathbf{x}) \mathbf{n}(\mathbf{x}), \mathbf{x} \in \Gamma\}$ is the perturbed interface, $D_{\varepsilon}^{-}$is the interior part of $\Gamma_{\varepsilon}$ and $D_{\varepsilon}^{+}:=D \backslash \overline{D_{\varepsilon}^{-}}$, and $\kappa(\mathbf{x}) \in \mathscr{A}$ is the normal variation. Moreover, we denote by $u_{\varepsilon}^{-}$(resp. $u_{\varepsilon}^{+}$) the perturbed solution's restriction on $D_{\varepsilon}^{-}\left(\operatorname{resp} . D_{\varepsilon}^{+}\right)$.

The first order shape derivative of the elliptic interface problem is defined formally by the pointwise limit

$$
\mathrm{d} u(\mathbf{x}):=\mathrm{d} u[\kappa](\mathbf{x})=\lim _{\varepsilon \rightarrow 0} \frac{u_{\varepsilon}(\mathbf{x})-u(\mathbf{x})}{\varepsilon}, \quad \mathbf{x} \in\left(D^{-} \cap D_{\varepsilon}^{-}\right) \cup\left(D^{+} \cap D_{\varepsilon}^{+}\right) .
$$

It is characterized by the following lemma.

Lemma 3.1. Under the Assumptions 2.1-2.3, the shape derivative $\mathrm{d} u:=\mathrm{d} u[\kappa]$ exists and satisfies the following EIP with nonhomogeneous jump conditions:

$$
\begin{aligned}
-\nabla \cdot(\alpha \nabla \mathrm{d} u) & =0 & & \text { in } D^{-} \cup D^{+}, \\
{[\mathrm{d} u] } & =-\kappa\left[\frac{\partial u}{\partial \mathbf{n}}\right] & & \text { on } \Gamma, \\
{\left[\alpha \frac{\partial \mathrm{d} u}{\partial \mathbf{n}}\right] } & =\nabla_{\Gamma} \cdot\left(\kappa[\alpha] \nabla_{\Gamma} u\right) & & \text { on } \Gamma, \\
\mathrm{d} u & =0 & & \text { on } \partial D .
\end{aligned}
$$

Here, $\nabla_{\Gamma}, \nabla_{\Gamma} \cdot$ denote, respectively, the surface gradient and the surface divergence operators which are defined by $\nabla_{\Gamma} v:=\nabla v-(\nabla v \cdot \mathbf{n}) \mathbf{n}$ for a scalar function $v$, and $\nabla_{\Gamma} \cdot \mathbf{v}:=\nabla \cdot \mathbf{v}-((\nabla \mathbf{v}) \mathbf{n}) \cdot \mathbf{n}$ for a vector field $\mathbf{v}$.

Proof. The plan of the proof is as follows. We take the difference of the respective variational formulations of the original problem (3.1)-(3.4) and of the perturbed problem (3.5)-(3.8) and let tend $\varepsilon \rightarrow 0$ to arrive at the variational formulation which belongs to the EIP (3.9)-(3.12) of the shape gradient.

The variational formulations of the original PDE (3.1)-(3.4) and of the perturbed problem (3.5)-(3.8) read as follows: Seek $u, u_{\varepsilon} \in H_{0}^{1}(D)$ such that

$$
\int_{D^{-}} \alpha^{-} \nabla u^{-} \cdot \nabla v \mathrm{~d} \mathbf{x}+\int_{D^{+}} \alpha^{+} \nabla u^{+} \cdot \nabla v \mathrm{~d} x=\int_{D} f v \mathrm{~d} \mathbf{x}
$$


and

$$
\int_{D_{\varepsilon}^{-}} \alpha^{-} \nabla u_{\varepsilon}^{-} \cdot \nabla v \mathrm{~d} \mathbf{x}+\int_{D_{\varepsilon}^{+}} \alpha \nabla u_{\varepsilon}^{+} \cdot \nabla v \mathrm{~d} \mathbf{x}=\int_{D} f v \mathrm{~d} \mathbf{x}
$$

for all $v \in H_{0}^{1}(D)$.

Subtracting (3.13) from (3.14), dividing by $\varepsilon$ on both sides and taking the limit $\varepsilon \rightarrow 0$, we obtain (see [39], Chap. 2 and [34], Chap. 4 for the details), the variational formulation of the shape gradient $\mathrm{d} u$ :

$$
\int_{D^{-}} \alpha^{-} \nabla \mathrm{d} u^{-} \cdot \nabla v \mathrm{~d} \mathbf{x}+\int_{D^{+}} \alpha^{+} \nabla \mathrm{d} u^{+} \cdot \nabla v \mathrm{~d} \mathbf{x}+\int_{\Gamma} \kappa\left(\alpha^{-} \nabla u^{-}-\alpha^{+} \nabla u^{+}\right) \cdot \nabla v \mathrm{~d} \mathbf{x}=0
$$

where we used the notation $\mathrm{d} u^{+}:=\left.\mathrm{d} u\right|_{D} ^{-}$and $\mathrm{d} u^{-}:=\left.\mathrm{d} u\right|_{D^{-}}$. Testing (3.15) by $\phi \in C_{0}^{\infty}\left(D^{+}\right)$and $\phi \in C_{0}^{\infty}\left(D^{-}\right)$, respectively, we arrive at the $\mathrm{PDE}(3.9)$.

The flux jump condition (3.11) follows by first testing (3.15) with a smooth function $\psi \in C^{\infty}\left(\mathbb{R}^{d}\right)$ on $\Gamma$ and then employing the flux jump condition (3.3) on $\Gamma$. This yields

$$
\alpha^{-} \frac{\partial \mathrm{d} u^{-}}{\partial \mathbf{n}}-\alpha^{+} \frac{\partial \mathrm{d} u^{+}}{\partial \mathbf{n}}=\nabla_{\Gamma} \cdot\left(\left(\alpha^{-}-\alpha^{+}\right) \kappa \nabla_{\Gamma} u\right)
$$

by using the tangential Green formula (cf. [14]) on the surface of co-dimension one, namely

$$
\int_{\Gamma} \nabla_{\Gamma} f \cdot \mathbf{v} \mathrm{d} s+\int_{\Gamma} f \nabla_{\Gamma} \cdot \mathbf{v} \mathrm{d} s=0
$$

where $f$ is a scalar function on the surface and $\mathbf{v}$ is a tangential vector field on the surface.

For $\psi \in C^{\infty}\left(\mathbb{R}^{d}\right)$, the jump condition (3.6) implies for the perturbed problem (3.5)-(3.8) that

$$
\int_{\Gamma_{\varepsilon}}\left(u_{\varepsilon}^{-}-u_{\varepsilon}^{+}\right) \psi \mathrm{d} s=0
$$

Pulling the integral back from $\Gamma_{\varepsilon}$ to $\Gamma$, taking the derivative with respect to $\varepsilon$ on both sides of this equality and evaluating it at $\varepsilon=0$, we obtain, by the same argument as in [14], equation (4.16), that

$$
\begin{aligned}
\int_{\Gamma}\left(\mathrm{d} u^{-}-\mathrm{d} u^{+}\right) \psi \mathrm{d} s & =-\int_{\Gamma} \kappa\left[\frac{\partial\left(\left(u^{-}-u^{+}\right) \psi\right)}{\partial \mathbf{n}}+(d-1) \mathcal{H}\left(u^{-}-u^{+}\right) \psi\right] \mathrm{d} s \\
& =-\int_{\Gamma} \kappa\left[\frac{\partial u}{\partial \mathbf{n}}\right] \psi \mathrm{d} s
\end{aligned}
$$

where $\mathcal{H}$ is the mean curvature of the interface $\Gamma$. Note that we inserted the jump condition (3.2) in the first equality to simplify this expression. The second equality finally implies the jump condition (3.10) due to the arbitrariness of $\psi$. This completes the proof.

Remark 3.2. It is worth noting that the shape gradient $\mathrm{d} u$ is in general not in $H_{0}^{1}(D)$ since it may be discontinuous along the interface $\Gamma$.

With the shape derivative and the Assumptions 2.1-2.3 at hand, we obtain for all $0 \leq \varepsilon \leq \varepsilon_{0}$ the pointwise first order shape-Taylor expansion

$$
u_{\varepsilon}(\mathbf{x})=u(\mathbf{x})+\varepsilon \mathrm{d} u[\kappa](\mathbf{x})+c(\mathbf{x}) \varepsilon^{2}, \quad \mathbf{x} \in D \backslash U_{\varepsilon_{0}}(\Gamma)
$$

where $U_{\varepsilon_{0}}(\Gamma)$ denotes the $\varepsilon_{0}$-tube region

$$
U_{\varepsilon_{0}}(\Gamma):=\left\{\mathbf{y}=\mathbf{x}+t \varepsilon_{0} \mathbf{n}(\mathbf{x}), t \in[-1,1], \mathbf{x} \in \Gamma\right\} .
$$


The constant $c(\mathbf{x})$ in (3.16) depends on the function $u$, the jump size $[a]$, and the distance of the point $\mathbf{x}$ to the interface, but it is bounded in $\kappa$ since we supposed $\|\kappa\|_{C^{2,1}(\Gamma, \mathbb{R})} \leq 1$. Assuming a compact subset $K \Subset D \backslash U_{\varepsilon_{0}}(\Gamma)$, we thus obtain from (3.16) the global first order shape-Taylor expansion

$$
u_{\varepsilon}(\mathbf{x})=u(\mathbf{x})+\varepsilon \mathrm{d} u[\kappa](\mathbf{x})+\mathcal{O}\left(\varepsilon^{2}\right), \quad \mathbf{x} \in K \Subset D \backslash U_{\varepsilon_{0}}(\Gamma) .
$$

\section{Statistics OF RANDOM SOLUTiOns}

In this section, we study the statistics of random solutions to SEIPs in terms of that of random interfaces through the perturbation method. The main idea behind the perturbation approach for the stochastic PDE is the matched asymptotic principle, which involves expanding all the random fields under concern around the expectation of the random interface via Taylor series expansion with a given small perturbation $\varepsilon$ and retaining terms up to high order, mostly second or third order. For a sufficiently small $\varepsilon_{0}>0$, we can develop a random version shape-Taylor expansion of random solutions to (2.1)-(2.4) with resort to the shape gradient, which is formulated in the following lemma.

Lemma 4.1. Under Assumptions 2.1-2.3, the random solution to (2.1)-(2.4) admits the following first order shape-Taylor expansion:

$$
u(\mathbf{x}, \omega)=\bar{u}(\mathbf{x})+\varepsilon \mathrm{d} u[\kappa(\omega)](\mathbf{x})+\mathcal{O}\left(\varepsilon^{2}\right)
$$

for all $\mathbf{x} \Subset K \subset D \backslash U_{\varepsilon_{0}}(\Gamma), P$-a.s. $\omega \in \Omega$. Here, $\bar{u}$ is the deterministic solution to the elliptic problem with the fixed reference interface $\Gamma$ and $\mathrm{d} u[\kappa(\omega)](\mathbf{x})$ is the associated shape derivative, given by the EIP (3.9)-(3.12) with $\kappa(\mathbf{x})$ being replaced by $\kappa(\mathbf{x}, \omega)$ and $D^{ \pm}$by $D^{ \pm}(\omega)$.

Proof. Applying the shape-Taylor expansion (3.18) for an arbitrary, fixed realization $\kappa(\omega), \omega \in \Omega$, yields in view of $\kappa(\omega) \in \mathcal{A}$ and Assumptions 2.1-2.3 its stochastic counterpart (4.1).

Now we can approximate the deterministic statistics of the random solution of (2.1)-(2.4) with the help to (4.1). More precisely, the mean field and the variance of random solutions can be approximated by their deterministic surrogates with respect to the reference interface in terms of certain approximation orders of the perturbation amplitude.

The following theorem addresses the semi-discrete approximation to the mean field in the stochastic dimension.

Theorem 4.2. The expectation $\mathbb{E}_{u}(\mathbf{x})$ of the random solutions to (2.1)-(2.4) can be approximated, up to the second order in terms of the perturbation amplitude $\varepsilon$, by solving the deterministic PDE (3.1)-(3.4) with respect to the reference interface $\Gamma$. More precisely, it holds that

$$
\mathbb{E}_{u}(\mathbf{x})=\bar{u}(\mathbf{x})+\mathcal{O}\left(\varepsilon^{2}\right), \quad \mathbf{x} \in K \Subset D \backslash U_{\varepsilon_{0}}(\Gamma),
$$

where $\bar{u}$ is the deterministic solution to the EIP (3.1)-(3.4) with the reference interface $\Gamma$.

Proof. We use the shape-Taylor expansion and obtain

$$
\mathbb{E}_{u}(\mathbf{x})=\bar{u}(\mathbf{x})+\varepsilon \mathbb{E}(\mathrm{d} u[\kappa(\omega)](\mathbf{x}))+\mathcal{O}\left(\varepsilon^{2}\right) .
$$

Since the expectation operator $\mathbb{E}$ is linear, we can take the expectation on both sides of the stochastic version of the PDE (3.9)-(3.12) of the shape derivative $\mathrm{d} u[\kappa(\omega)](\mathbf{x})$, which yields, in view of $\mathbb{E}_{\kappa}(\mathbf{x})=0$, the equation

$$
\mathbb{E}(\mathrm{d} u[\kappa(\omega)](\mathbf{x}))=0 .
$$

From this, the desired claim follows. 
As we will see, we can approximate $\operatorname{Var}_{u}(\mathbf{x})$ by deterministically solving the tensor product PDE of $\operatorname{Cor}_{\mathrm{d} u}(\mathbf{x}, \mathbf{y})$ on the product domain $D \times D \subset \mathbb{R}^{2 d}$. Denote by $\llbracket \cdot \rrbracket$ the tensor-product of the jump operator which is given for any two-point correlation function $w(\mathbf{x}, \mathbf{y})$ by

$$
\llbracket w(\mathbf{x}, \mathbf{y}) \rrbracket=\lim _{\substack{D^{-} \ni \mathbf{x \rightarrow T} \\ D^{-} \ni \mathbf{y} \rightarrow \Gamma}} w(\mathbf{x}, \mathbf{y})-\lim _{\substack{D^{-} \ni \mathbf{x} \rightarrow \Gamma \\ D^{+} \ni \mathbf{y} \rightarrow \Gamma}} w(\mathbf{x}, \mathbf{y})-\lim _{\substack{D^{+} \ni \mathbf{x \rightarrow} \rightarrow \Gamma \\ D^{-} \ni \mathbf{y} \rightarrow \Gamma}} w(\mathbf{x}, \mathbf{y})+\lim _{\substack{D^{+} \ni \mathbf{x \rightarrow \rightarrow} \\ D^{+} \ni \mathbf{y} \rightarrow \Gamma}} w(\mathbf{x}, \mathbf{y}) .
$$

Then, the tensor product boundary value problem for $\operatorname{Cor}_{\mathrm{d} u}(\mathbf{x}, \mathbf{y})$ can be stated as follows:

- Tensor-product-domain PDE:

$$
\left(\nabla_{\mathbf{x}} \otimes \nabla_{\mathbf{y}}\right) \cdot\left(\alpha(\mathbf{x}) \alpha(\mathbf{y})\left(\nabla_{\mathbf{x}} \otimes \nabla_{\mathbf{y}}\right) \operatorname{Cor}_{\mathrm{d} u}(\mathbf{x}, \mathbf{y})\right)=0,
$$

for all $(\mathbf{x}, \mathbf{y}) \in D^{ \pm} \times D^{ \pm}$;

- Interface-domain PDE:

$$
\begin{array}{r}
-\nabla_{\mathbf{y}} \cdot\left(\alpha(\mathbf{y}) \nabla_{\mathbf{y}}\left[\operatorname{Cor}_{\mathrm{d} u}(\mathbf{x}, \mathbf{y})\right]_{\mathbf{x}}\right)=0 \\
-\nabla_{\mathbf{y}} \cdot\left(\alpha(\mathbf{y}) \nabla_{\mathbf{y}}\left[\alpha(\mathbf{x}) \frac{\partial \operatorname{Cor}_{\mathrm{d} u}}{\partial \mathbf{n}_{\mathbf{x}}}(\mathbf{x}, \mathbf{y})\right]_{\mathbf{x}}\right)=0
\end{array}
$$

for all $(\mathbf{x}, \mathbf{y}) \in \Gamma \times D^{ \pm}$;

- Domain-interface PDE:

$$
\begin{array}{r}
-\nabla_{\mathbf{x}} \cdot\left(\alpha(\mathbf{x}) \nabla_{\mathbf{x}}\left[\operatorname{Cor}_{\mathrm{d} u}(\mathbf{x}, \mathbf{y})\right]_{\mathbf{y}}\right)=0 \\
-\nabla_{\mathbf{x}} \cdot\left(\alpha(\mathbf{x}) \nabla_{\mathbf{x}}\left[\alpha(\mathbf{y}) \frac{\partial \operatorname{Cor}_{\mathrm{d} u}}{\partial \mathbf{n}_{\mathbf{y}}}(\mathbf{x}, \mathbf{y})\right]_{\mathbf{y}}\right)=0
\end{array}
$$

for all $(\mathbf{x}, \mathbf{y}) \in D^{ \pm} \times \Gamma$;

- DD tensor interface condition:

$$
\llbracket \operatorname{Cor}_{\mathrm{d} u}(\mathbf{x}, \mathbf{y}) \rrbracket=\operatorname{Cor}_{\kappa}(\mathbf{x}, \mathbf{y})\left[\frac{\partial \bar{u}}{\partial \mathbf{n}}(\mathbf{x})\right]\left[\frac{\partial \bar{u}}{\partial \mathbf{n}}(\mathbf{y})\right],
$$

for all $(\mathbf{x}, \mathbf{y}) \in \Gamma \times \Gamma$;

- DN and ND tensor interface condition:

$$
\begin{aligned}
& \llbracket \alpha(\mathbf{y}) \frac{\partial \operatorname{Cor}_{\mathrm{d} u}}{\partial \mathbf{n}_{\mathbf{y}}}(\mathbf{x}, \mathbf{y}) \rrbracket=-\nabla_{\Gamma, \mathbf{y}} \cdot\left(\left[\frac{\partial \bar{u}}{\partial \mathbf{n}}(\mathbf{x})\right][\alpha(\mathbf{y})] \operatorname{Cor}_{\kappa}(\mathbf{x}, \mathbf{y}) \nabla_{\Gamma} \bar{u}(\mathbf{y})\right) \\
& \llbracket \alpha(\mathbf{x}) \frac{\partial \operatorname{Cor}_{\mathrm{d} u}}{\partial \mathbf{n}_{\mathbf{x}}}(\mathbf{x}, \mathbf{y}) \rrbracket=-\nabla_{\Gamma, \mathbf{x}} \cdot\left(\left[\frac{\partial \bar{u}}{\partial \mathbf{n}}(\mathbf{y})\right][\alpha(\mathbf{x})] \operatorname{Cor}_{\kappa}(\mathbf{x}, \mathbf{y}) \nabla_{\Gamma} \bar{u}(\mathbf{x})\right)
\end{aligned}
$$

for all $(\mathbf{x}, \mathbf{y}) \in \Gamma \times \Gamma$;

- NN tensor interface condition:

$$
\llbracket \alpha(\mathbf{x}) \alpha(\mathbf{y}) \frac{\partial^{2} \operatorname{Cor}_{\mathrm{d} u}}{\partial \mathbf{n}_{\mathbf{x}} \partial \mathbf{n}_{\mathbf{y}}}(\mathbf{x}, \mathbf{y}) \rrbracket=\left(\nabla_{\Gamma, \mathbf{x}} \otimes \nabla_{\Gamma, \mathbf{y}}\right) \cdot\left([\alpha(\mathbf{x})][\alpha(\mathbf{y})] \operatorname{Cor}_{\kappa}(\mathbf{x}, \mathbf{y}) \nabla_{\Gamma} \bar{u}(\mathbf{x}) \nabla_{\Gamma} \bar{u}(\mathbf{y})\right),
$$

for all $(\mathbf{x}, \mathbf{y}) \in \Gamma \times \Gamma$;

- Tensor-product boundary condition:

$$
\operatorname{Cor}_{\mathrm{d} u}(\mathbf{x}, \mathbf{y})=0,
$$

for all $(\mathbf{x}, \mathbf{y}) \in \partial(D \times D)$.

The solution $\operatorname{Cor}_{\mathrm{d} u}(\mathbf{x}, \mathbf{y})$ to the tensorized PDE (4.2)-(4.11) is crucial for the approximation of the variance of the random solution to (2.1)-(2.4). One can either solve it by the sparse grid technique from $[19,23]$ or employ the algorithm which we propose in the next section.

The second theorem studies the approximation of the variance of random solutions. 
Theorem 4.3. The variance $\operatorname{Var}_{u}(\mathbf{x})$ of the random solutions to (2.1)-(2.4) can be approximated, up to the third order in terms of the perturbation amplitude $\varepsilon$, by solving the deterministic tensor-product PDE (4.2)-(4.11). More precisely, it holds

$$
\operatorname{Var}_{u}(\mathbf{x})=\varepsilon^{2} \operatorname{Var}_{\mathrm{d} u}(\mathbf{x})+\mathcal{O}\left(\varepsilon^{3}\right), \quad \mathbf{x} \in K \Subset D \backslash U_{\varepsilon_{0}}(\Gamma),
$$

where

$$
\begin{aligned}
\operatorname{Var}_{\mathrm{d} u}(\mathbf{x}) & =\operatorname{Var}(\mathrm{d} u[\kappa(\omega)](\mathbf{x})) \\
& =\left.\operatorname{Cor}(\mathrm{d} u[\kappa(\omega)](\mathbf{x}), \mathrm{d} u[\kappa(\omega)](\mathbf{y}))\right|_{\mathbf{y}=\mathbf{x}} \\
& =\left.\operatorname{Cor}_{\mathrm{d} u}(\mathbf{x}, \mathbf{y})\right|_{\mathbf{y}=\mathbf{x}},
\end{aligned}
$$

and $\operatorname{Cor}_{\mathrm{d} u}(\mathbf{x}, \mathbf{y})$ is the solution to (4.2)-(4.11).

Proof. Consider the two random variables $X$ and $Y$ with finite second moments. Then, on the one hand, we find the identity

$$
\operatorname{Var}(a+b X+c Y)=b^{2} \operatorname{Var}(X)+2 b c \operatorname{Covar}(X, Y)+c^{2} \operatorname{Var}(Y),
$$

On the other hand, the following inequality holds due to the Cauchy-Schwarz inequality:

$$
\operatorname{Covar}(X, Y) \leq \sqrt{\operatorname{Var}(X) \cdot \operatorname{Var}(Y)} .
$$

Combining (4.13) and (4.14) with the shape-Taylor expansion (4.1), we have

$$
\begin{aligned}
\operatorname{Var}_{u}(\mathbf{x}) & =\varepsilon^{2} \operatorname{Var}(\mathrm{d} u[\kappa(\omega)](\mathbf{x}))+\mathcal{O}\left(\varepsilon^{4}\right)+\sqrt{\operatorname{Var}(\mathrm{d} u[\kappa(\omega)](\mathbf{x}))} \mathcal{O}\left(\varepsilon^{3}\right) \\
& =\varepsilon^{2} \operatorname{Var}(\mathrm{d} u[\kappa(\omega)](\mathbf{x}))+\mathcal{O}\left(\varepsilon^{3}\right) .
\end{aligned}
$$

Hence the proof is completed.

Remark 4.4. The relative error of the approximation of the variance is of order $\varepsilon$. In [11], it is shown that, under the present assumptions, even higher order moments are approximated with relative error $\varepsilon$. Moreover, there are also given precise estimates on the constants hidden by the present $\mathcal{O}$-notation.

\section{ERROR ESTIMATES AND IMPLEMENTATION}

In this section, error estimates will be carried out for the fully discrete approximations of the mean field and two-point correlation function of random solutions in terms of both the mesh size in the physical domain and the perturbation amplitude in the stochastic dimension. We propose the efficient computation of the random solution's variance by a low-rank approximation via the pivoted Cholesky decomposition. The evaluation of shape derivates can be avoided if finite differences are used.

In the sequel, we employ a shape regular and interface-resolved triangulation for the finite element discretization of the EIP system (3.1)-(3.4) to achieve optimal convergence rates for the deterministic PDE. By "interface-resolved" we mean that the vertices of elements around the interface lie exactly on the interface $(c f .[10,31])$. By the use of parametric mappings, we are even endowed with hierarchical finite element spaces amenable for the sparse tensor approximation of the two-point correlation function (cf. [19]).

\subsection{Computing the approximate expectation}

We have presented the discussion of the semi-discrete approximation in the stochastic dimension of the mean field and two-point correlation function in the continuous case by the asymptotic analysis in terms of the perturbation amplitude. Now, we develop the finite element discretization to obtain a fully discrete approximation. 
In particular, we derive an error estimate in terms of both, the finite element mesh size of the triangulation and the perturbation amplitude.

Assume that the triangulation $\left\{\mathscr{T}_{h}\right\}_{h>0}$ is a sequence of quasi-uniform and shape-regular triangulations with the mesh size $h$, and let $\left\{V_{h}\right\}_{h>0}$ be the corresponding finite element spaces over the given triangulations. It is noted that the finite elements used in the numerical analysis can be piecewise linear or of arbitrary degree provided that the underlying triangulation resolves the interface. In our particular implementation, we use continuous piecewise linear finite elements. Armed with the finite element spaces, we can obtain the discrete finite element approximation $\bar{u}_{h}$ to $\bar{u}$ by solving the variational formulation of (3.1)-(3.4) with respect to the reference interface $\Gamma$ in the finite dimensional space $V_{h}$.

The discretization error of the deterministic solution $\bar{u}$ can be quantified by the follow lemma adapted from [31], Theorem 4.1 with a little modification.

Theorem 5.1. Let $\left\{\mathscr{T}_{h}\right\}_{h>0},\left\{V_{h}\right\}_{h>0}$ be a family of interface-resolved triangulations and the associated finite element spaces (cf. [19,31]). Let $\bar{u}_{h}$ be the deterministic finite element solution corresponding to the deterministic solution $\bar{u}(\mathbf{x})$ of the elliptic problem (3.1)-(3.4) with respect to the reference interface $\Gamma$. Then, for $s=0,1$, there holds that

$$
\left\|\bar{u}-\bar{u}_{h}\right\|_{H^{s}(D)} \leq C h^{2-s}\|\bar{u}\|_{H^{2}\left(D^{-}\right) \cup H^{2}\left(D^{+}\right)},
$$

where $H^{2}\left(D^{-}\right) \cup H^{2}\left(D^{+}\right)$is the standard broken Sobolev space equipped by the norm

$$
\|\cdot\|_{H^{2}\left(D^{-}\right) \cup H^{2}\left(D^{+}\right)}:=\sqrt{\|\cdot\|_{H^{2}\left(D^{-}\right)}^{2}+\|\cdot\|_{H^{2}\left(D^{+}\right)}^{2}} .
$$

On $K \Subset D \backslash U_{\varepsilon_{0}}(\Gamma)$, there holds for the expectation $\mathbb{E}_{u}$ of the random solution to the SEIP (2.1)-(2.4)

$$
\left\|\mathbb{E}_{u}(\mathbf{x})-\bar{u}_{h}(\mathbf{x})\right\|_{H^{s}(K)} \leq C\left(\varepsilon^{2}+h^{2-s}\right)\|\bar{u}\|_{H^{2}\left(D^{-}\right) \cup H^{2}\left(D^{+}\right)} .
$$

Proof. The estimate (5.1) readily follows from $[10,31]$ for at least $C^{2}$-smooth interfaces. The estimate for the expectation is derived from combining Theorem 4.2, (5.1) and the following triangle inequality

$$
\left\|\mathbb{E}_{u}-\bar{u}_{h}\right\|_{H^{s}(K)} \leq\left\|\mathbb{E}_{u}-\bar{u}\right\|_{H^{s}(K)}+\left\|\bar{u}-\bar{u}_{h}\right\|_{H^{s}(K)} .
$$

\subsection{Computing the two-point correlation function}

\subsubsection{Computing local shape derivatives}

Notice that the PDE for the local shape derivative $\mathrm{d} u=\mathrm{d} u[\kappa]$ is not amenable for a naive finite element discretization due to the non-homogeneous Dirichlet jump condition. Here, we use the offset function technique to convert the original PDE (3.9)-(3.12) into two systems.

We shall start with the week formulation of the interface problem. We seek the function $\mathrm{d} u=\mathrm{d} u[\kappa] \in$ $H^{1}\left(D^{-}\right) \cup H^{1}\left(D^{+}\right)$such that

$$
\begin{gathered}
\int_{D^{-}} \alpha^{-} \nabla \mathrm{d} u \nabla v \mathrm{~d} \mathbf{x}+\int_{D^{+}} \alpha^{+} \nabla \mathrm{d} u \nabla v \mathrm{~d} \mathbf{x}=-\int_{\Gamma} \kappa[\alpha] \nabla_{\Gamma} \bar{u} \nabla_{\Gamma} v \mathrm{~d} s, \quad v \in H_{0}^{1}(D) \\
\text { and }[\mathrm{d} u]=-\kappa\left[\frac{\partial \bar{u}}{\partial \mathbf{n}}\right] \text { on } \Gamma, \quad \mathrm{d} u=0 \text { on } \partial D .
\end{gathered}
$$

Note that we used integration by parts to remove the surface divergence in the Neumann jump.

To solve this variational problem, we make the ansatz $\mathrm{d} u=\mathrm{d} u_{\text {hom }}+\mathrm{d} u_{\text {off }}$, where the offset function $\mathrm{d} u_{\text {off }}^{-}=$ $\mathrm{d} u_{\text {off }}^{-}[\kappa] \in H^{1}\left(D^{-}\right)$solves the inhomogeneous Dirichlet problem

$$
\begin{gathered}
\int_{D^{-}} \alpha^{-} \nabla \mathrm{d} u_{\text {off }}^{-} \nabla v \mathrm{~d} \mathbf{x}=0, \quad v \in H_{0}^{1}\left(D^{-}\right) \\
\text {and } \mathrm{d} u_{\text {off }}^{-}=-\kappa\left[\frac{\partial \bar{u}}{\partial \mathbf{n}}\right] \text { on } \Gamma .
\end{gathered}
$$


We extend this function trivially to $D^{+}$by setting $\mathrm{d} u_{\text {off }}^{+}:=0$. If we subtract $\mathrm{d} u_{\text {off }}$ from $\mathrm{d} u$, we are led to seek $\mathrm{d} u_{\text {hom }}=\mathrm{d} u_{\text {hom }}[\kappa] \in H_{0}^{1}(D)$ such that

$$
\begin{gathered}
\int_{D^{-}} \alpha^{-} \nabla \mathrm{d} u_{\mathrm{hom}} \nabla v \mathrm{~d} \mathbf{x}+\int_{D^{+}} \alpha^{+} \nabla \mathrm{d} u_{\mathrm{hom}} \nabla v \mathrm{~d} \mathbf{x}=-\int_{D^{-}} \alpha^{-} \nabla \mathrm{d} u_{\mathrm{off}} \nabla v \mathrm{~d} \mathbf{x}-\int_{\Gamma} \kappa[\alpha] \nabla_{\Gamma} \bar{u} \nabla_{\Gamma} v \mathrm{~d} s, \quad v \in H_{0}^{1}(D) \\
\text { and }\left[\mathrm{d} u_{\mathrm{hom}}\right]=0 \text { on } \Gamma, \quad \mathrm{d} u_{\mathrm{hom}}=0 \text { on } \partial D
\end{gathered}
$$

The computation of $\mathrm{d} u_{\text {off }}$ and $\mathrm{d} u_{\text {hom }}$ via (5.3) and (5.4), respectively, by a interface-resolved finite element method is straightforward if we restrict the finite element spaces $V_{j}$ onto $D^{-}$and $D$, respectively.

Theorem 5.2. Assume $\left\{\mathscr{T}_{h}\right\}_{h>0},\left\{V_{h}\right\}_{h>0}$ as specified in Subsection 5.1. For given $\kappa \in C^{2,1}(\Gamma, \mathbb{R})$, let $\mathrm{d} u=$ $\mathrm{d} u[\kappa]$ denote the shape derivative given by (3.9)-(3.12) and let $\mathrm{d} u_{h}$ be its finite element approximation via combining the respective finite element solutions from (5.3) and (5.4). Then, for $s=0,1$, there holds that

$$
\begin{aligned}
\left\|\mathrm{d} u-\mathrm{d} u_{h}\right\|_{H^{s}\left(D^{-}\right) \cup H^{s}\left(D^{+}\right)} & \leq C h^{2-s}\left\{\left\|\kappa\left[\frac{\partial \bar{u}}{\partial \mathbf{n}}\right]\right\|_{H^{3 / 2}(\Gamma)}+\left\|\nabla_{\Gamma} \cdot\left(\kappa[\alpha] \nabla_{\Gamma} \bar{u}\right)\right\|_{H^{1 / 2}(\Gamma)}\right\} \\
& \leq C h^{2-s}\|\bar{u}\|_{H^{3}\left(D^{-}\right) \cup H^{3}\left(D^{+}\right)}
\end{aligned}
$$

provided that the given data are sufficiently smooth.

Proof. It is obvious that the successive solution of (5.3) and (5.4) yields the solution of (5.2). Thus, from $[7,8]$, it follows for $s=0,1$ the estimate

$$
\left\|\mathrm{d} u-\mathrm{d} u_{h}\right\|_{H^{s}\left(D^{-}\right) \cup H^{s}\left(D^{+}\right)} \leq C h^{2-s}\left\{\left\|\kappa\left[\frac{\partial \bar{u}}{\partial \mathbf{n}}\right]\right\|_{H^{3 / 2}(\Gamma)}+\left\|\nabla_{\Gamma} \cdot\left(\kappa[\alpha] \nabla_{\Gamma} \bar{u}\right)\right\|_{H^{1 / 2}(\Gamma)}\right\} .
$$

Finally, in view of

$$
\left\|\kappa\left[\frac{\partial \bar{u}}{\partial \mathbf{n}}\right]\right\|_{H^{3 / 2}(\Gamma)} \lesssim\|\bar{u}\|_{H^{3}\left(D^{-}\right) \cup H^{3}\left(D^{+}\right)}
$$

and

$$
\left\|\nabla_{\Gamma} \cdot\left(\kappa[\alpha] \nabla_{\Gamma} \bar{u}\right)\right\|_{H^{1 / 2}(\Gamma)} \lesssim\|\bar{u}\|_{H^{5 / 2}(\Gamma)} \lesssim\|\bar{u}\|_{H^{3}\left(D^{-}\right) \cup H^{3}\left(D^{+}\right)},
$$

we conclude also the last estimate.

Remark 5.3. In the above error estimate (5.5), we have not taken into account that we have only access to the numerical approximation $\bar{u}_{h}$ of $\bar{u}$. This approximation induces an additional consistency error. It is of the order $\mathcal{O}(h)$ due to

$$
\left\|\bar{u}-\bar{u}_{h}\right\|_{H^{1 / 2}(\Gamma)} \lesssim\left\|\bar{u}-\bar{u}_{h}\right\|_{H^{1}(D)} \lesssim h\left\|\bar{u}-\bar{u}_{h}\right\|_{H^{2}\left(D^{-}\right) \cup H^{2}\left(D^{+}\right)}
$$

and

$$
\left\|\left[\frac{\partial \bar{u}}{\partial \mathbf{n}}\right]-\left[\frac{\partial \bar{u}_{h}}{\partial \mathbf{n}}\right]\right\|_{H^{-1 / 2}(\Gamma)} \lesssim\left\|\bar{u}-\bar{u}_{h}\right\|_{H^{1}(D)} \lesssim h\left\|\bar{u}-\bar{u}_{h}\right\|_{H^{2}\left(D^{-}\right) \cup H^{2}\left(D^{+}\right)} .
$$

Hence, due to the consistency error of the order $\mathcal{O}(h)$, we can only expect the reduced convergence rate $\mathcal{O}(h)$ instead of $\mathcal{O}\left(h^{2}\right)$ for the approximation of the shape derivative. 


\subsubsection{Low-rank approximation}

We can proceed in two different ways to compute the two-point correlation function Cor $_{\mathrm{d} u}$ given by (4.2)(4.11). The first way is a direct solution in the sparse tensor product space as considered in e.g. [19,23]. The over-all computational complexity is then essentially linear (i.e., linear up to logarithmic terms). However, the implementation of this approach for the interface problem under consideration is nontrivial.

The second way, which is much simpler to implement, consists in computing a low-rank approximation

$$
\operatorname{Cor}_{\kappa} \approx \sum_{i=1}^{m} \kappa_{i} \otimes \kappa_{i}
$$

of the two-point correlation function of $\kappa(\omega)$ in the full tensor product space $V_{h} \otimes V_{h}$. With such a low-rank approximation at hand we have

$$
\operatorname{Cor}_{\mathrm{d} u} \approx \sum_{i=1}^{m} \mathrm{~d} u\left[\kappa_{i}\right] \otimes \mathrm{d} u\left[\kappa_{i}\right]
$$

due to the linearity of the mapping $\kappa \mapsto \mathrm{d} u[\kappa]$. Hence, it suffices to solve (5.2) for all $\kappa_{i}$.

Assume that $\mathrm{Cor}_{\kappa} \in C(\Gamma \times \Gamma)$ is continuous, then the discrete version of the low-rank approximation (5.6) corresponds to the low-rank decomposition

$$
\mathbf{C} \approx \mathbf{C}_{m}=\sum_{i=1}^{m} \boldsymbol{\ell}_{i} \ell_{i}^{T}
$$

of the matrix

$$
\mathbf{C}=\left[\operatorname{Cor}_{\kappa}\left(\mathbf{x}_{i}, \mathbf{x}_{j}\right)\right]_{i, j} \in \mathbb{R}^{n \times n}
$$

where $\left\{\mathbf{x}_{i}\right\}$ are the nodes of the finite element mesh.

The best low-rank approximation (5.6) with respect to $L^{2}(\Gamma \times \Gamma)$ is given by the spectral decomposition whose computation requires the knowledge of the eigenpairs $\left(\varphi_{i}, \lambda_{i}\right)$ of the integral operator

$$
\left(\mathcal{K}_{\kappa} u\right)(\mathbf{x}):=\int_{\Gamma} \operatorname{Cor}_{\kappa}(\mathbf{x}, \mathbf{y}) u(\mathbf{y}) \mathrm{d} \mathbf{y}, \quad \mathbf{x} \in \Gamma .
$$

The decay of the eigenvalues $\left\{\lambda_{i}\right\}$ and thus the rank $m$ depends heavily on the smoothness of Cor $_{\kappa}$. Related decay rates have been proven in $[18,38]$.

We use here the pivoted Cholesky decomposition to compute a low-rank approximation of Cor $_{\kappa}$ as proposed in [21]. It is a purely algebraic approach which is quite simple to implement, see Algorithm 1. It produces a lowrank approximation to $\mathbf{C}$ for any given precision $\varepsilon>0$ where the approximation error is rigorously controlled in the trace norm. A rank- $m$ approximation is computed in $\mathcal{O}\left(m^{2} n\right)$ operations, if $n$ denotes the number of nodes $\left\{\mathbf{x}_{i}\right\}$ of the finite element mesh. Exponential convergence rates in $m$ are proven under the assumption that the eigenvalues of $\mathbf{C}$ exhibit a sufficiently fast exponential decay, see [21]. Nevertheless, numerical experiments show that, in general, the pivoted Cholesky decomposition converges optimally in the sense that the rank $m$ is uniformly bounded by the number of terms required for the spectral decomposition of Cor $_{\kappa}$ to get the error $\varepsilon$.

Having the low-rank decomposition (5.6) of the interface perturbation's two-point correlation function at hand, the complexity to compute the shape derivative's two-point correlation (5.7) is at least $\mathcal{O}(m n)$. Therefore, $\log$-linear complexity in $n$ can only be achieved if $m \sim \log n$ which requires an exponential decay of the spectral decomposition of the two-point correlation $\mathrm{Cor}_{\kappa}$. This, however, can only be shown in the case that Cor $_{\kappa}$ is analytical (cf. [38]). In contrast to that, the sparse grid approach from [19,23] produces approximations in essentially linear complexity also for two-point correlation functions of finite smoothness. We refer the reader to [18] for a more extensive comparison of the spectral decomposition and the sparse grid approach. 


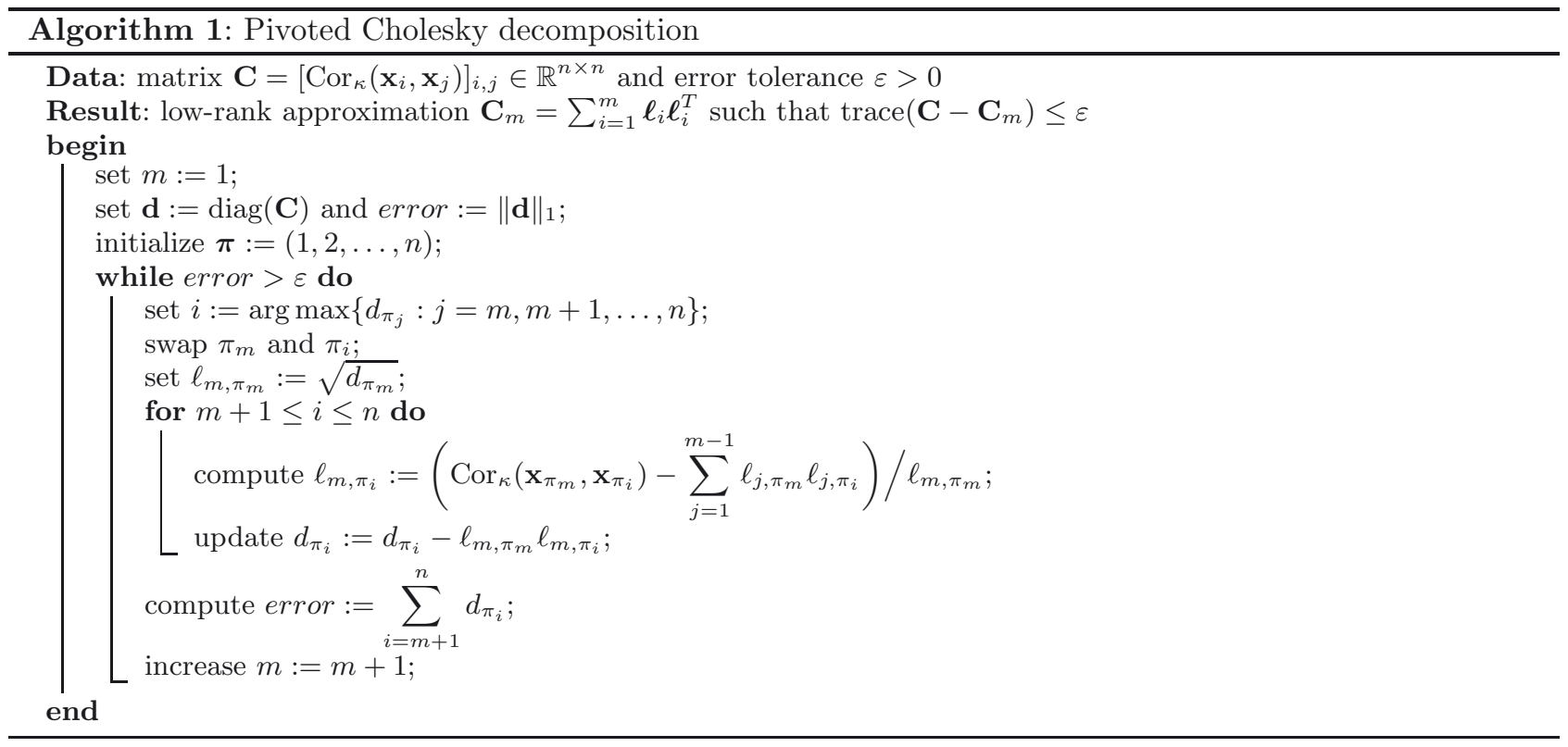

\subsubsection{Non-intrusive approach}

Instead of computing the local shape derivative $\mathrm{d} u\left[\kappa_{i}\right]$, we can approximate it by a finite difference. This means that, for a small $h>0$, we replace (5.7) by

$$
\operatorname{Cor}_{\mathrm{d} u} \approx \frac{1}{h^{2}} \sum_{i=1}^{m}\left(u_{\Gamma\left(I+h \kappa_{i}\right)}-\bar{u}\right) \otimes\left(u_{\Gamma\left(I+h \kappa_{i}\right)}-\bar{u}\right),
$$

where $u_{\Gamma\left(I+h \kappa_{i}\right)}$ denotes the solution of the interface problem (2.1)-(2.4) with respect to the perturbed interface

$$
\Gamma\left(I+h \kappa_{i}\right):=\left\{\mathbf{x}+h \kappa_{i}(\mathbf{x}) \mathbf{n}(\mathbf{x}): \mathbf{x} \in \Gamma\right\} .
$$

Thus, it is neither necessary to explicitly know the local shape derivative nor to implement it. In fact, besides the computation of the approximate expectation $\bar{u}$, we need then only the $m$ "samples" $u_{\Gamma\left(I+h \kappa_{i}\right)}$ to calculate the approximate variance. This therefore constitutes an extremely fast non-intrusive algorithm in the quantification of domain uncertainties. The drawback is that we need to construct for each sample $u_{\Gamma\left(I+h \kappa_{i}\right)}$ a new interfaceresolved triangulation in order not to lose accuracy.

\section{NumERICAL EXPERIMENTS AND DISCUSSION}

\subsection{Deterministic vs. Monte Carlo approach}

We present some numerical tests to demonstrate our theoretical predictions. Let $D:=(-1,1)^{2}$ be a square of edge length 2 and let

$$
\Gamma:=\left\{\mathbf{x} \in \mathbb{R}^{2}:\|\mathbf{x}\|=1 / 2\right\}
$$

be a circular interface which subdivides $D$ into the interior domain $D^{-}$and the exterior domain $D^{+}$. In the exterior domain $D^{+}$the diffusion coefficient is set to $\alpha^{+}:=1$. In the interior domain $D^{-}$we consider $\alpha^{-}:=2$ in our first and $\alpha^{-}:=10$ in our second example. 
We parametrize the interface $\Gamma$ by polar coordinates

$$
\bar{\gamma}:[0,2 \pi] \rightarrow \Gamma, \quad s \mapsto \bar{\gamma}(s):=\frac{1}{2}\left[\begin{array}{c}
\cos (s) \\
\sin (s)
\end{array}\right] .
$$

Correspondingly, the stochastic interface $\Gamma_{\varepsilon}(\omega)$ can be expressed via the perturbed parametrization

$$
\gamma(s, \omega):=\bar{\gamma}(s)+\varepsilon \kappa(s, \omega)\left[\begin{array}{c}
\cos (s) \\
\sin (s)
\end{array}\right]
$$

Herein, we assume that the stochastic interface perturbation is given by

$$
\kappa(s, \omega):=\sum_{k=0}^{5}\left\{a_{k}(\omega) \cos (k s)+b_{k}(\omega) \sin (k s)\right\}
$$

with stochastic coefficients $a_{k}(\omega)$ and $b_{k}(\omega)$ being equally distributed in $[-1,1]$ and mutually stochastically independent. This leads to the two-point correlation function

$$
\operatorname{Cor}_{\kappa}(s, t)=\frac{1}{3} \sum_{k=0}^{5}\{\cos (k s) \cos (k t)+\sin (k s) \sin (k t)\} .
$$

For our numerical experiments we choose $\varepsilon=0.02$. Even though $\varepsilon$ is small the perturbation is considerably large since the norm $\|\kappa(\omega)\|_{C^{2,1}([0,2 \pi])}$ might become large. In particular, the boundary points vary up to about \pm 0.15 around the related unperturbed point on the reference interface.

We determine first the expectation and the variance of random solutions to this SEIP by a MC method, using $M=10000$ samples. The triangulation has to be reconstructed for each sample in order to resolve the interface exactly. In order to compute the sample mean and variance, we interpolate the solution to a fixed quadrangular grid with $65 \times 65$ nodes. The approximate expectation and variance are depicted in the first row of Figure 2 for $\alpha^{-}:=2$ and Figure 3 for $\alpha^{-}:=10$.

In the second row of Figures 2 and 3 the approximate expectation and variance of our deterministic algorithm is depicted. To ensure a fair comparison, we interpolated the finite element solutions on the same rectangular mesh as the MC solutions. Notice that the pivoted Cholesky decomposition computes an exact rank-11 approximation of the two-point correlation (6.1) since it is of finite rank 11. This means that only 11 shape derivatives need to be determined to compute the approximate variance.

In the last row of Figures 2 and 3, we plotted the differences of expectations and variances of both algorithms. We see that the deterministic approach produces quite accurate approximations except in the direct neighborhood of the reference interface, as predicted by our theory.

Finally, we shall validate the asymptotic behavior in the perturbation parameter $\varepsilon$ given by Theorems 4.2 and 4.3. At the interface, we remove the tube $U_{\varepsilon_{0}}(\Gamma)$ of size $\varepsilon_{0}=0.2(c f$. (3.17)) to define the domain $K:=$ $D \backslash U_{\varepsilon_{0}}(\Gamma)$. On this domain $K$ and for the choices $\alpha^{-}:=2,10,50$ (as above we set $\alpha^{+}:=1$ ), we compare for $0 \leq$ $\varepsilon \leq 0.02$ the approximate mean and variance of the deterministic approach with a related MC simulation. Note that our choice of $K$ ensures that the interfaces of the samples in the MC method are always contained in $U_{\varepsilon_{0}}(\Gamma)$. In particular, the assumptions of Lemma 4.1 hold. We displayed the $L^{2}(K)$-errors versus the perturbation parameter $\varepsilon$ in Figure 4 (in fact, the graph is the mean of three independent simulations). In case of the expectation (left plot), we observe indeed that the error between the deterministic method and the MC method is of the order $\mathcal{O}\left(\varepsilon^{2}\right)$ (indicated by the dashed line). In case of the variance (right plot), we find that the error between the deterministic method and the MC method is of the order $\mathcal{O}\left(\varepsilon^{3}\right)$ (also indicated by the dashed line). The results are thus in good agreement with our theoretical findings on the asymptotic behaviour in $\varepsilon$. On the other hand, for fixed $\varepsilon$, we observe that the error increases if $\alpha^{-}$and thus the jump size $[\alpha]$ increases. This means that the constant in (3.16) (as already mentioned there) and thus the method is not robust in the jump size $[\alpha]$. 
Expectation and variance of the Monte Carlo simulation
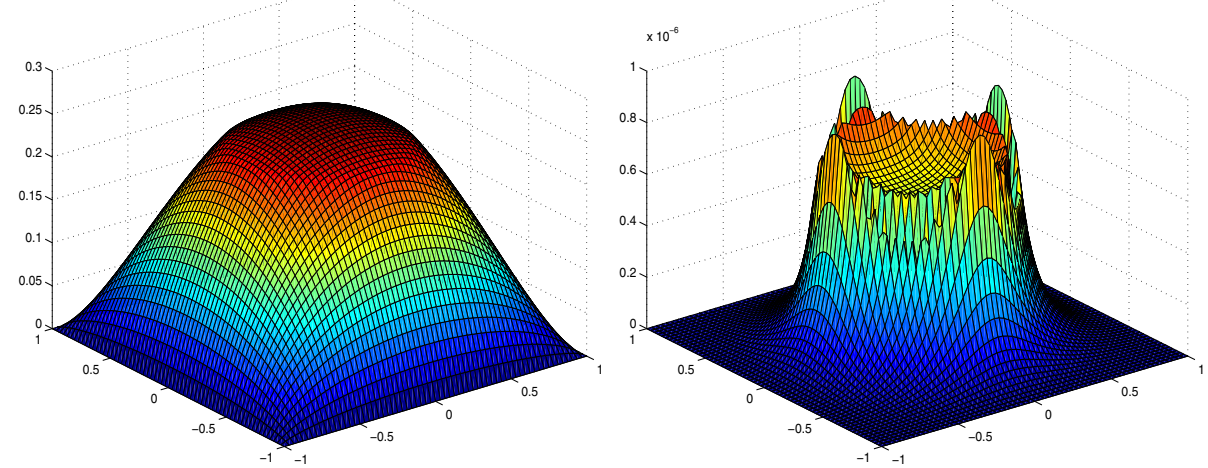

Expectation and variance of the perturbation approach

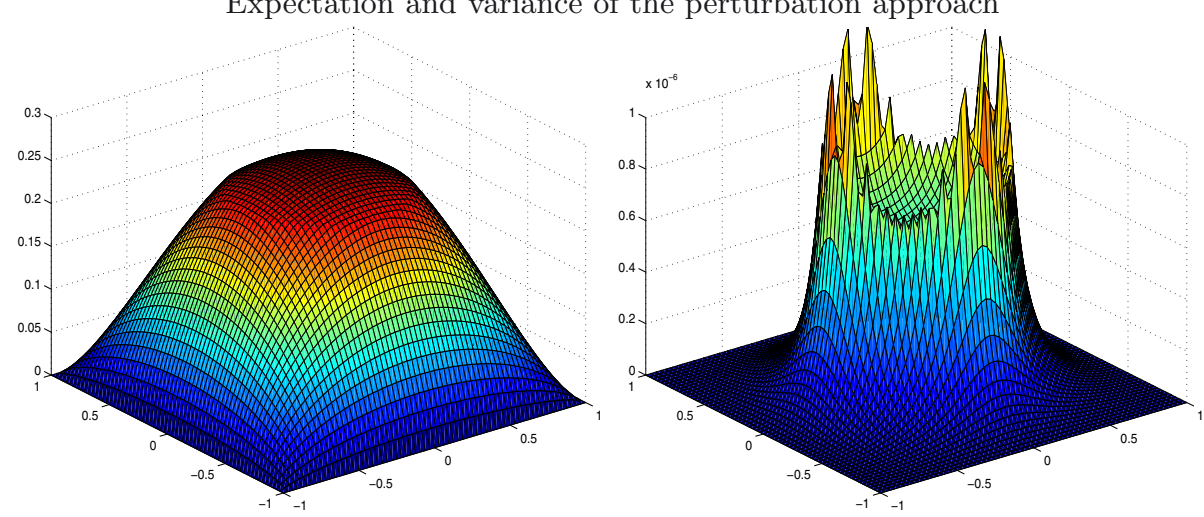

Difference of the expectations and variances
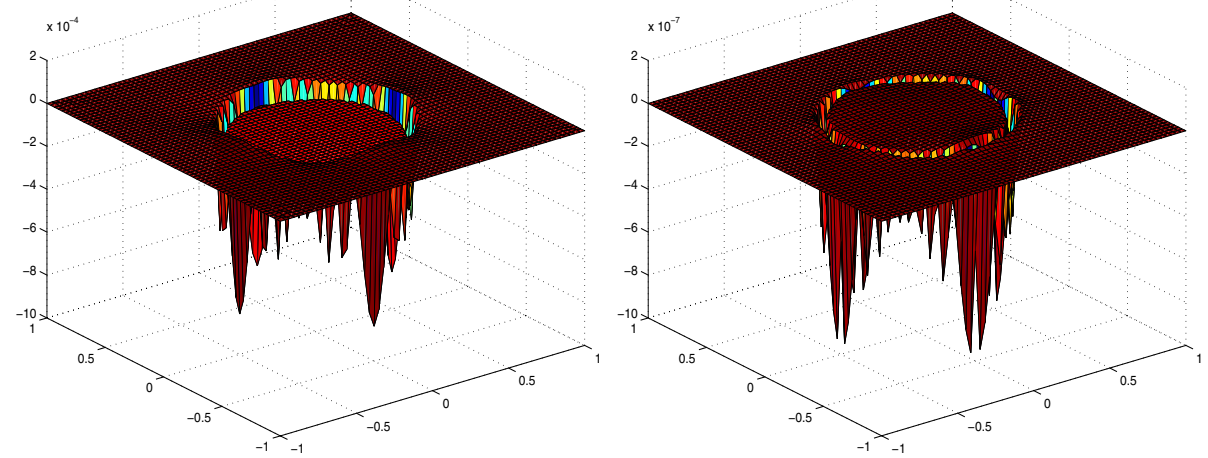

Figure 2. Approximate expectation and variation in case of $\alpha^{-}=2$ and $\varepsilon=0.02$.

\subsection{Non-intrusive approach}

Our next example is concerned with the comparison of the shape derivative based approach (5.7) and the non-intrusive approach (5.10). The domain $D$ and the interface $\Gamma$ are chosen as in the previous example while the associated coefficients are set to $\alpha^{-}:=2$ and to $\alpha^{+}=1$. The stochastic perturbation of the interface is assumed to exhibit the Gaussian two-point correlation

$$
\operatorname{Cor}_{\kappa}=\exp \left(-\sigma\|\mathbf{x}-\mathbf{y}\|^{2}\right)
$$

where we consider the choices $\sigma=10,100,1000$. 
Expectation and variance of the Monte Carlo simulation
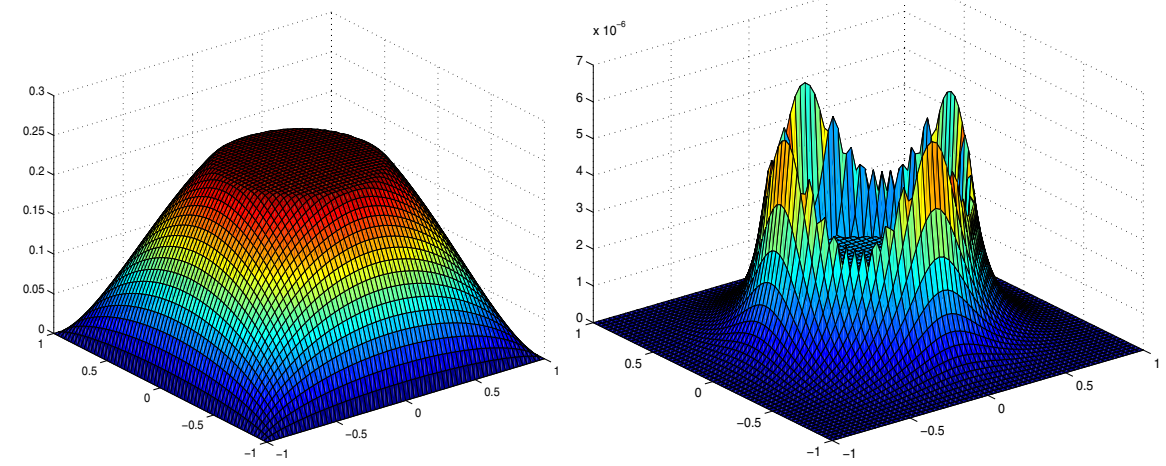

Expectation and variance of the perturbation approach

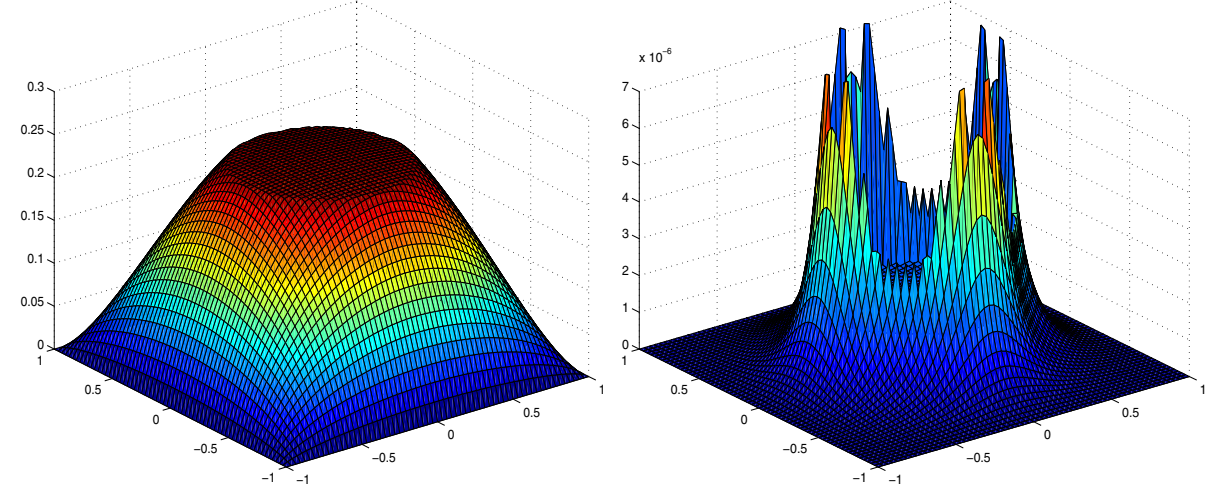

Difference of the expectations and variances
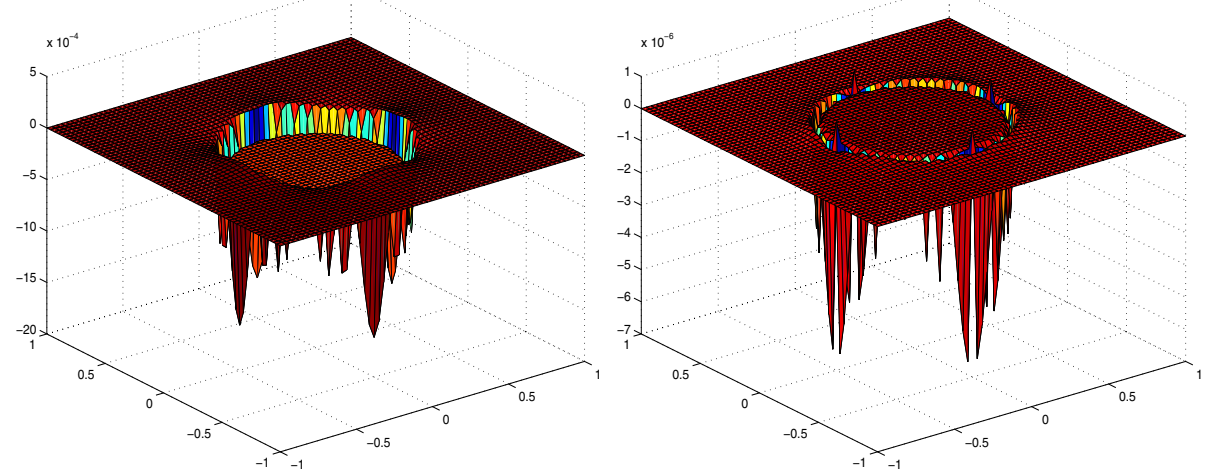

FiguRE 3. Approximate expectation and variation in case of $\alpha^{-}=10$ and $\varepsilon=0.02$.

We again use the pivoted Cholesky decomposition (Algorithm 1) to compute the discrete low-rank decomposition (5.8) with a trace error of $\varepsilon=0.001$. It has the rank $m=17$ if $\sigma=10$, the rank $m=54$ if $\sigma=100$, and the rank $m=171$ if $\sigma=1000$. The step size $h$ in the finite differences of (5.10) is chosen as $h=0.001$. The resulting approximate variances are plotted in Figures $5-7$. As these plots demonstrate, the non-intrusive approach (5.10) produces reasonable approximations of the solution's variance while the implementation is extremely simple. Especially, we observe that the influence of the stochastic interface becomes more localized as $\sigma$ increases, i.e., if the correlation length of the interface perturbation decreases. 

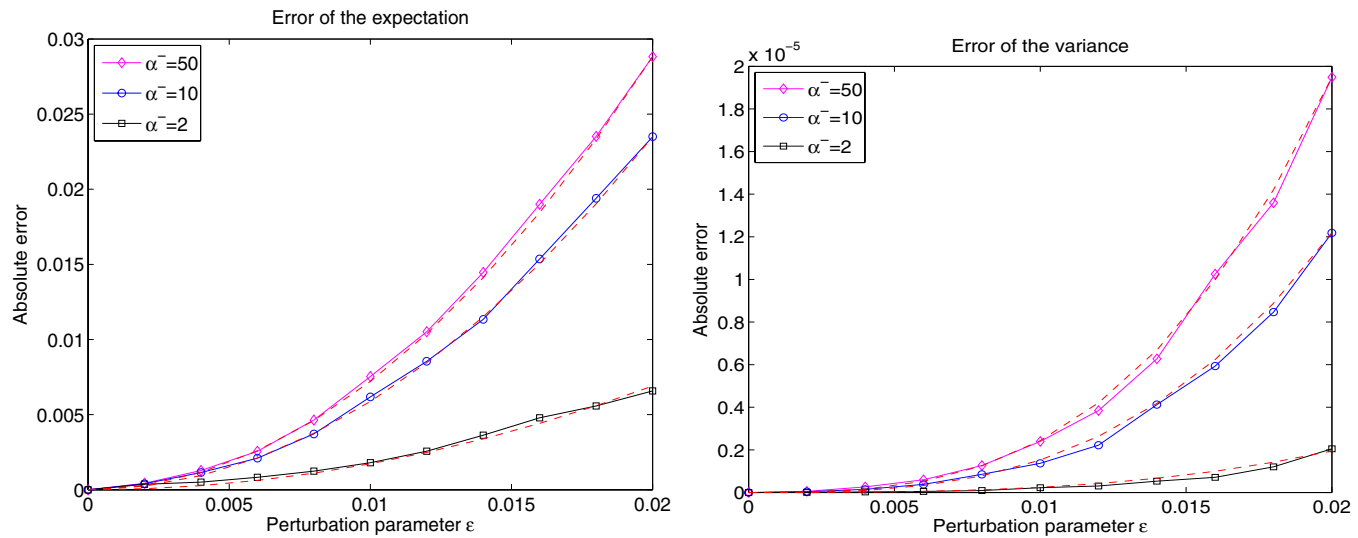

FiguRe 4. Asymptotic behaviour in the perturbation parameter $\varepsilon$ in case of the expectation (left plot) and in case of the variance (right plot).
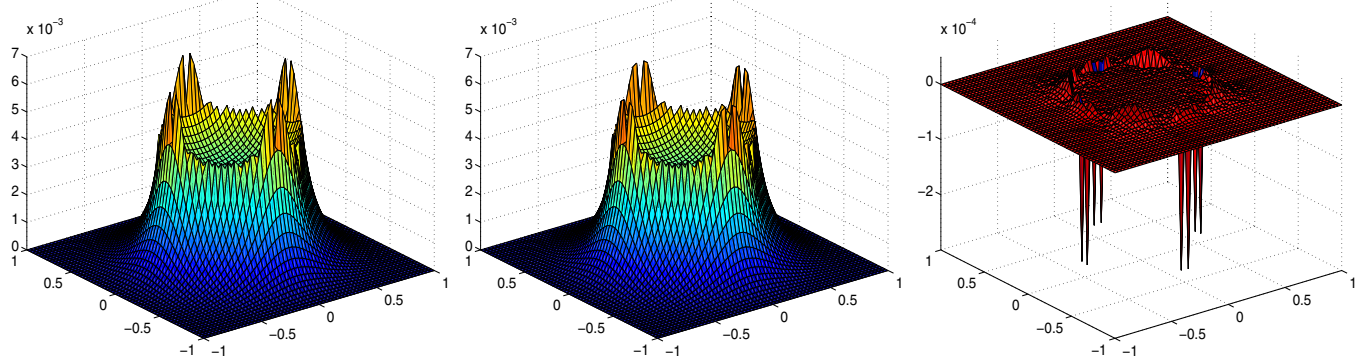

Figure 5. Approximate variations (left: via shape derivative / middle: via finite difference / right: difference between the approaches) in case of the Gaussian kernel with $\sigma=10$.
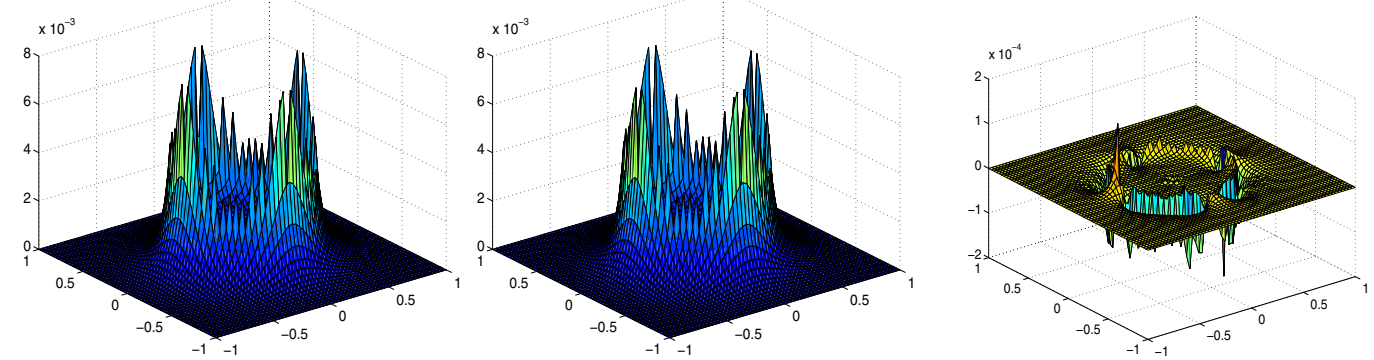

FiguRE 6. Approximate variations (left: via shape derivative / middle: via finite difference / right: difference between the approaches) in case of the Gaussian kernel with $\sigma=100$. 

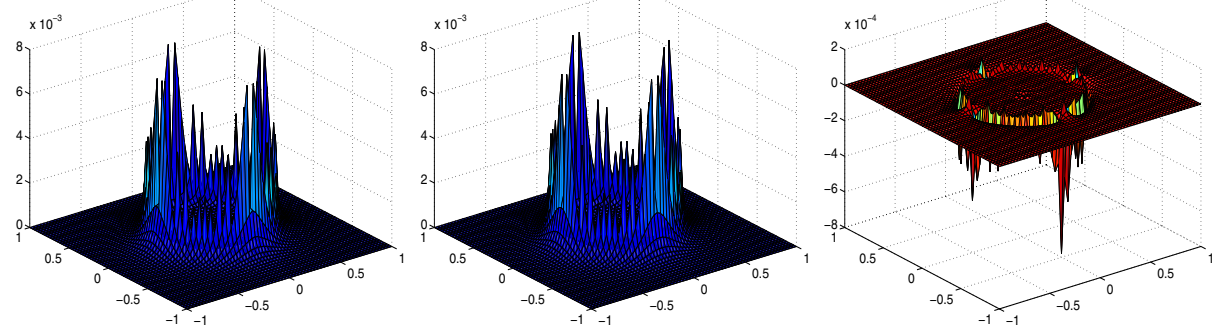

FiguRE 7. Approximate variations (left: via shape derivative / middle: via finite difference / right: difference between the approaches) in case of the Gaussian kernel with $\sigma=1000$.

Acknowledgements. The authors would like to thank the anonymous referees for their careful reading of the manuscript and their insightful comments and suggestions which helped us improve the manuscript significantly. The second author is greatly indebted to Prof. Christoph Schwab from ETH Zürich for introducing him to this intriguing stochastic PDE field through attending his fascinating course in the fall semester of 2010 at ETH.

\section{REFERENCES}

[1] I. Babuška and P. Chatzipantelidis, On solving elliptic stochastic partial differential equations. Comput. Methods Appl. Mech. Engrg. 191 (2002) 4093-4122.

[2] I. Babuška, F. Nobile and R. Tempone, Worst case scenario analysis for elliptic problems with uncertainty. Numer. Math. 101 (2005) 185-219.

[3] I. Babuška, F. Nobile and R. Tempone, A stochastic collocation method for elliptic partial differential equations with random input data. SIAM J. Num. Anal. 45 (2007) 1005-1034.

[4] I. Babuška, R. Tempone and G.E. Zouraris, Galerkin finite element approximations of stochastic elliptic partial differential equations. SIAM J. Numer. Anal. 42 (2004) 800-825.

[5] A. Barth, C. Schwab and N. Zollinger, Multi-Level Monte Carlo Finite Element method for elliptic PDE's with stochastic coefficients. Numer. Math. 119 (2011) 123-161.

[6] V.I. Bogachev, Gaussian Measures, Mathematical Surveys and Monographs in vol. 62. AMS, Providence, RI (1998).

[7] J.H. Bramble and J.T. King, A finite element method for interface problems with smooth boundaries and interfaces. Adv. Comput. Math. 6 (1996) 109-138.

[8] J.W. Barrett and C.M. Elliott, Fitted and unfitted finite-element methods for elliptic equations with interfaces. IMA J. Numer. Anal. 7 (1987) 283-300.

[9] C. Canuto and T. Kozubek, A fictitious domain approach to the numerical solution of pdes in stochastic domains. Numer. Math. 107 (2007) 257-293.

[10] Z. Chen and J. Zou, Finite element methods and their convergence for elliptic and parabolic interface problems. Numer. Math. 79 (1998) 175-202.

[11] A. Chernov and C. Schwab, First order $k$-th moment finite element analysis of nonlinear operator equations with stochastic data. Math. Comput. To appear (2012).

[12] M.K. Deb, I.M. Babuska and J.T. Oden, Solution of stochastic partial differential equations using Galerkin finite element techniques. Comput. Methods Appl. Mech. Engrg. 190 (2001) 6359-6372.

[13] B.J. Debusschere, H.N. Najm, P.P. Pébay, O.M. Knio, R.G. Ghanem and O.P.L. Maître, Numerical challenges in the use of polynomial chaos representations for stochastic processes. SIAM J. Sci. Comput. 26 (2004) 698-719.

[14] M.C. Delfour and J.-P. Zolesio, Shapes and Geometries - Analysis, Differential Calculus, and Optimization. SIAM, Society for Industrial and Appl. Math., Philadelphia (2001).

[15] F.R. Desaint and J.-P. Zolésio, Manifold derivative in the Laplace-Beltrami equation. J. Functional Anal. 151 (1997) $234-269$.

[16] P. Frauenfelder, C. Schwab and R.A. Todor, Finite elements for elliptic problems with stochastic coefficients. Comput. Methods Appl. Mech. Engrg. 194 (2005) 205-228.

[17] R.G. Ghanem and P.D. Spanos, Stochastic finite elements: a spectral approach. Springer-Verlag (1991).

[18] M. Griebel and H. Harbrecht, Approximation of bivariate functions: singular value decomposition versus sparse grids. IMA J. Numer. Anal. To appear (2013).

[19] H. Harbrecht, A finite element method for elliptic problems with stochastic input data. Appl. Numer. Math. 60 (2010) $227-244$.

[20] H. Harbrecht, On output functionals of boundary value problems on stochastic domains. Math. Meth. Appl. Sci. 33 (2010) 91-102. 
[21] H. Harbrecht, M. Peters and R. Schneider, On the low-rank approximation by the pivoted Cholesky decomposition. Appl. Numer. Math. 62 (2012) 428-440.

[22] H. Harbrecht, R. Schneider and C. Schwab, Multilevel frames for sparse tensor product spaces. Numer. Math. 110 (2008) 199-220.

[23] H. Harbrecht, R. Schneider and C. Schwab, Sparse second moment analysis for elliptic problems in stochastic domains. Numer. Math. 109 (2008) 385-414.

[24] F. Hettlich and W. Rundell, The determination of a discontinuity in a conductivity from a single boundary measurement. Inverse Problems 14 (1998) 67-82.

[25] F. Hettlich and W. Rundell, Identification of a discontinuous source in the heat equation. Inverse Problems 17 (2001) 14651482 .

[26] K. Ito, K. Kunisch and Z. Li, Level-set function approach to an inverse interface problem. Inverse Problems 17 (2001) 12251242.

[27] J.B. Keller, Stochastic equations and wave propagation in random media. Proc. Symp. Appl. Math. in vol. 16. AMS, Providence, R.I. (1964) 145-170.

[28] M. Kleiber and T.D. Hien, The stochastic finite element method: basic perturbation technique and computer implementation. Wiley, Chichester (1992).

[29] P.E. Kloeden and E. Platen, Numerical solution of stochastic differential equations. Springer, Berlin 3rd ed. (1999).

[30] M. Ledoux and M. Talagrand, Probability in Banach Spaces. Isoperimetry and Processes. Springer, Berlin (1991).

[31] J. Li, J.M. Melenk, B. Wohlmuth and J. Zou, Optimal a priori estimates for higher order finite elements for elliptic interface problems. Appl. Numer. Math. 60 (2010) 19-37.

[32] Z. Li and K. Ito, The immersed interface method: numerical solutions of PDEs involving interfaces and irregular domains. SIAM, Society for Industrial and Appl. Math., Philadelphia (2006).

[33] H.G. Matthies and A. Keese, Galerkin methods for linear and nonlinear elliptic stochastic partial differential equations. Comput. Methods Appl. Mech. Engrg. 194 (2005) 1295-1331.

[34] O. Pironneau, Optimal Shape Design for Elliptic Systems. Springer, New York (1984).

[35] P. Protter, Stochastic Integration and Differential Equations: A New Approach. Springer, Berlin, 3rd ed. (1995).

[36] C. Schwab and R.A. Todor, Sparse finite elements for elliptic problems with stochastic loading. Numer. Math. 95 (2003) 707-734.

[37] C. Schwab and R.A. Todor, Sparse finite elements for stochastic elliptic problems - higher order moments. Comput. 71 (2003) 43-63.

[38] C. Schwab and R.A. Todor, Karhunen-Loéve approximation of random fields by generalized fast multipole methods. J. Comput. Phys. 217 (2006) 100-122.

[39] J. Sokolowski and J.-P. Zolesio, Introduction to Shape Optimization: Shape Sensitivity Analysis. Springer-Verlag (1992).

[40] T. von Petersdorff and C. Schwab, Sparse finite element methods for operator equations with stochastic data. Appl. Math. 51 (2006) 145-180.

[41] X. Wan, B. Rozovskii and G. E. Karniadakis, A stochastic modeling method based on weighted Wiener chaos and Malliavan calculus. PNAS 106 (2009) 14189-14194.

[42] J. Wloka, Partial Differential Equations. Cambridge University Press, Cambridge (1987).

[43] D. Xiu and G.E. Karniadakis, Modeling uncertainty in steady state diffusion problems via generalized polynomial chaos. Comput. Methods Appl. Mech. Engrg. 191 (2002) 4927-4948.

[44] D. Xiu and D.M. Tartakovsky, Numerical methods for differential equations in random domains. SIAM J. Scientific Comput. 28 (2006) 1167-1185. 